\section{(C) OPEN ACCESS}

\title{
Challenges in modelling the Charcot-Marie-Tooth neuropathies for therapy development
}

\author{
Manisha Juneja, ${ }^{1,2}$ Joshua Burns, ${ }^{3}$ Mario A Saporta, ${ }^{4}$ Vincent Timmerman ${ }^{1,2}$
}

- Additional material is

published online only. To view please visit the journal online (http://dx.doi.org/10.1136/ jnnp-2018-318834).

${ }^{1}$ Peripheral Neuropathy Research Group, University of Antwerp, Antwerp, Belgium ${ }^{2}$ Neurogenetics Labatory, Institute Born Bunge, Antwerp, Belgium

${ }^{3}$ University of Sydney, Faculty of Health Sciences \& Sydney Children's Hospitals Network, Sydney, New South Wales, Australia

${ }^{4}$ Department of Neurology, Leonard M. Miller School of Medicine, University of Miami, Miami, Florida, USA

\section{Correspondence to}

Dr Vincent Timmerman,

Peripheral Neuropathy Research Group, University of Antwerp, Antwerp 2610, Belgium; vincent.timmerman@ uantwerpen.be

Received 16 May 2018 Revised 18 June 2018 Accepted 19 June 2018 Published Online First 17 July 2018

Check for updates

(C) Author(s) (or their employer(s)) 2019. Re-use permitted under CC BY-NC. No commercial re-use. See rights and permissions. Published by BMJ.

To cite: Juneja M, Burns J, Saporta MA, et al. J Neurol Neurosurg Psychiatry

2019:90:58-67.

\section{ABSTRACT}

Much has been achieved in terms of understanding the complex clinical and genetic heterogeneity of CharcotMarie-Tooth neuropathy (CMT). Since the identification of mutations in the first CMT associated gene, PMP22, the technological advancement in molecular genetics and gene technology has allowed scientists to generate diverse animal models expressing monogenetic mutations that closely resemble the CMT phenotype. Additionally, one can now culture patient-derived neurons in a dish using cellular reprogramming and differentiation techniques. Nevertheless, despite the fact that finding a disease-causing mutation offers a precise diagnosis, there is no cure for CMT at present. This review will shed light on the exciting advancement in CMT disease modelling, the breakthroughs, pitfalls, current challenges for scientists and key considerations to move the field forward towards successful therapies.

\section{CHARCOT-MARIE-TOOTH DISEASE, A HETEROGENEOUS HEREDITARY MOTOR AND SENSORY NEUROPATHY}

Charcot-Marie-Tooth neuropathy (CMT) is the most common hereditary motor and sensory neuropathy with an estimated worldwide prevalence of 1 in $2500 .{ }^{1}$ The disease was named after the three neurologists, Jean-Martin Charcot, Pierre Marie and Howard Henry Tooth, who first described the disease in $1886 .{ }^{23} \mathrm{CMT}$ is a clinically and genetically heterogeneous disease, affecting both children and adults. Patients typically exhibit a slowly progressive and length-dependent degeneration of their peripheral nerves resulting in muscle weakness, atrophy in the feet and legs which extend later to the hands, causing reduced tendon reflexes and slight to moderate distal sensory impairment. ${ }^{4}$ Foot deformities like pes cavus (high-arched foot deformity) is among the frequently reported manifestations of CMT, with patients sometimes also exhibiting hearing loss and hip dysplasia. ${ }^{5}$ These and other additional symptoms that mark the different CMT subtypes may cause a significant decrease in the quality of life of affected individuals. $^{78}$

Based on electrophysiological criteria and the cell type affected, CMT is broadly classified into two major subgroups, CMT1 and CMT2. CMT1 is a demyelinating peripheral neuropathy affecting primarily the myelinating Schwann cells and is further characterised by slow nerve conduction velocities (NCV) $(<38 \mathrm{~m} / \mathrm{sin}$ patients). CMT2, on the other hand, is characterised by axonal degeneration and NCVs are within the normal $(>40-45 \mathrm{~m} / \mathrm{s})$ or occasionally in the mildly abnormal range (30-40 $\mathrm{m} / \mathrm{s}$ ). Compound muscle action potential (CMAP) amplitudes, reflecting the degree of axonal damage in motor nerves, are found to be reduced in patients with CMT2. ${ }^{9}$ However, it is important to note that axonal loss is a feature of both CMT2 and CMT1 and it is the major determinant of disability in patients with CMT, even when axonal loss is secondary to demyelination. To further complicate the phenotypic classification of CMT, intermediate forms (DI-CMT) which demonstrate overlapping electrophysiological and neuropathological features between CMT1 and CMT2, even within the same family, have been increasingly recognised in recent years. Mutations in over 120 genes have been found to be associated with the pathogenesis of CMT and related neuropathies, with most of them being unveiled in the past 25 years owing to the rapid advancement in molecular genetics and next generation sequencing technology. ${ }^{10}$ Mutations that cause CMT can affect genes that have specific functions in Schwann cells or neurons, but can also affect ubiquitously expressed genes. A list of CMT associated genes in conjunction with specific variant information can be found at the Inherited Neuropathy Variant Browser (INVB; https://hihg.med. miami.edu/neuropathybrowser). ${ }^{11}$ These mutations can be inherited in an autosomal-dominant, $\mathrm{X}$ linked or autosomal recessive manner and also include mitochondrial DNA mutations. Because of this heterogeneity, the current OMIM nomenclature used to classify CMT neuropathies in different subtypes became unclear and confusing. Therefore, a new CMT classification system has been recently proposed but it still needs general acceptance by clinicians and scientists (table 1). ${ }^{12}$ Important to note is that overlapping phenotypes exist; patients exhibiting demyelination of peripheral nerves usually show signs of secondary axonal degeneration, as noted above. ${ }^{5}$ In addition, the overlap in the genetic and phenotypic spectrum of CMT with distal hereditary motor neuropathies (dHMN), hereditary sensory and autonomic neuropathies, spinal muscular atrophy (SMA), hereditary spastic paraplegias (HSP), mitochondrial and other neuromuscular disorders further increase the complexity.

Functional studies on the different CMT mutations have been extensively pursued over the years. The wide array of neuropathy genes identified so far and their relevance in terms of structural and functional aspects in the human peripheral nervous system, have been well illustrated by Weis et al, 2017. ${ }^{13}$ Great mechanistic diversity has been 
Table 1 Animal models used for emulating CMT disease

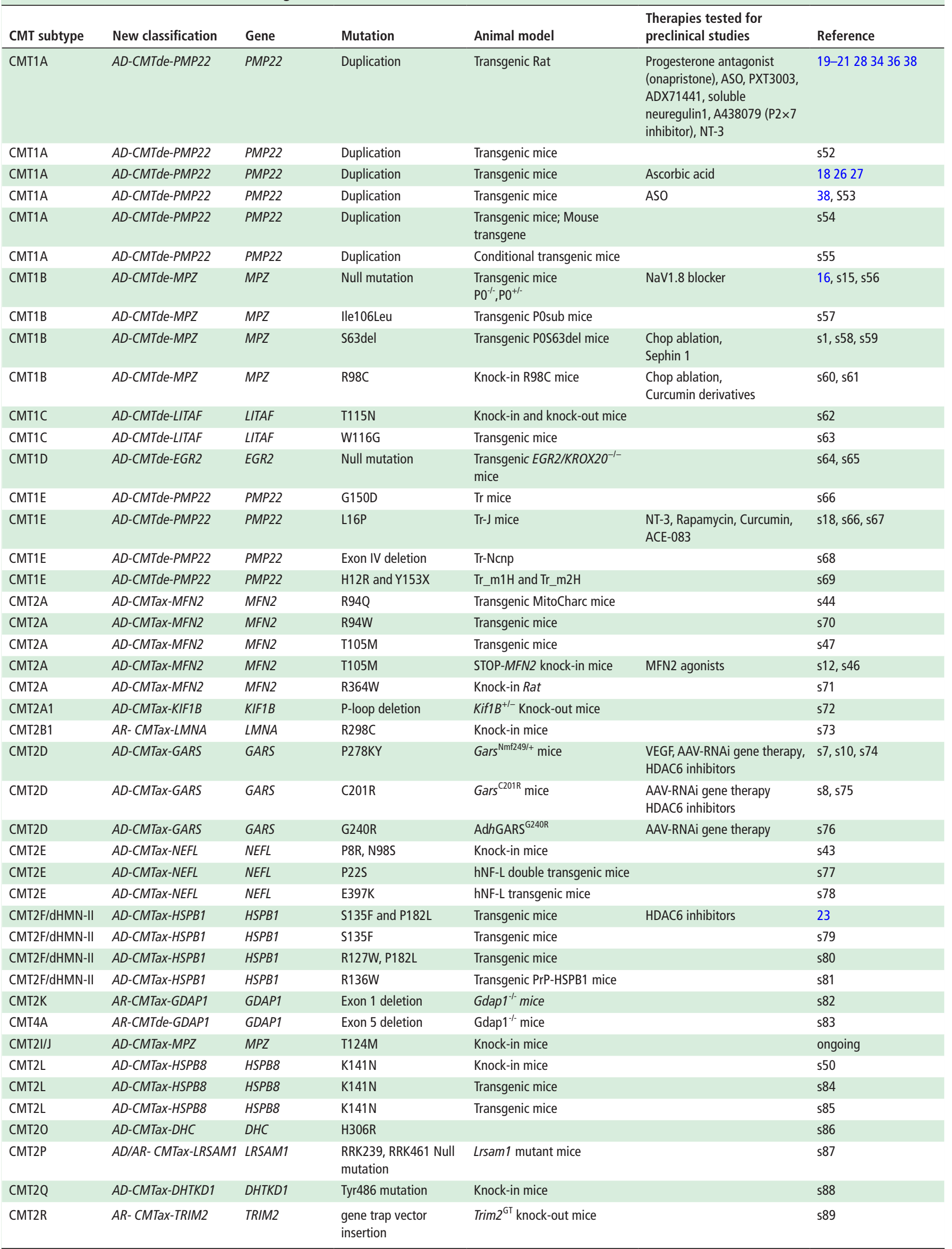




\begin{tabular}{|c|c|c|c|c|c|c|}
\hline CMT subtype & New classification & Gene & Mutation & Animal model & $\begin{array}{l}\text { Therapies tested for } \\
\text { preclinical studies }\end{array}$ & Reference \\
\hline DI-CMTC & AD-CMTin-YARS & YARS & E196K mutation & AdhYARS ${ }^{\text {E196K/ChAT mice }}$ & & s90 \\
\hline CMTX1 & $X L-C M T i n-G J B$ & $G J B / C \times 32$ & G12S, S26L & Transgenic mice & CamKII inhibitors & s91, s92 \\
\hline CMTX1 & $X L-C M T i n-G J B$ & $\begin{array}{l}\text { GJB1/ } \\
\text { CX32 }\end{array}$ & Exon2 deletion & Gjb1 ${ }^{\text {tm1Kwi }}$ knock-out mice & Intrathecal Vector Delivery & s93, s94 \\
\hline
\end{tabular}

ASO, antisense oligonucleotide; CMT, Charcot-Marie-Tooth;VEGF, vascular endothelial growth factor.

observed between the different CMT-causal genes while explicit links are missing. Unfortunately, a curative treatment for CMT is not yet available and major breakthroughs in the field are yet to be achieved. This is due to three major obstacles: (1) an enormous genetic heterogeneity (over 1500 CMT causal point mutations, including the $1.4 \mathrm{Mb}$ CMT1A duplication) and overlapping phenotypes associated with the disease, (2) a limited number of patients per genotype thereby attracting limited interest from researchers and pharmaceutical companies and (3) difficulties in translating the preclinical studies from rodents and cellular models to human clinical trials.

In this review, we will summarise the developments in the research models published to date for dissecting molecular pathways in CMT and to identify drug targets and biomarkers of disease. Importantly, we will highlight the translational efforts made so far, the lessons learnt from failures and pinpoint the possibilities of improving the drug discovery process for CMT. The core of drug discovery lies in identifying the right drug target. Considering the current estimated probability of success for bringing an orphan drug (excluding oncology indications) to the market is a mere $13.6 \%,{ }^{14}$ this might be a good time to reflect about possible reasons behind the failures, redefining new strategies to identify biologically relevant molecular targets and specific drugs. We hope that this review will encourage researchers to redesign their strategies, to build up a strong molecular basis for identifying disease mechanisms and drug targets collectively aiming to improve the clinical success rate for treating such rare and heterogeneous disorders.

\section{DISEASE MODELLING OF CMT NEUROPATHIES USING RODENT MODELS: FROM PATHOMECHANISMS TO THERAPEUTIC INSIGHTS}

The knowledge about the nature of CMT has greatly expanded owing to the successful generation of disease models mimicking the patient phenotype. The first report describing the naturally occurring point mutation in a myelin gene in the Trembler mouse dates back to $1992 .{ }^{15} \mathrm{~A}$ few years later, the first genetically modified animal model with a quintessential phenotype and clinical characteristic of CMT was created. ${ }^{16}$ Murine therapeutic models for CMT1 and CMT4 have been previously reviewed by Fledrich et al, 2012. ${ }^{17}$ Since then, numerous CMT rodent models have been successfully generated (table 1) and paved the way for delineating the pathomechanisms and initiating preclinical studies to validate different therapeutic strategies. Reducing peripheral myelin protein 22 (PMP22) gene expression was one of the first attempts to treat CMT1A caused by a tandem duplication harbouring PMP22 on human chromosome 17. This resulted in the identification and evaluation of ascorbic acid, onapristone and neurotrophin as potential therapeutics. ${ }^{18-21}$ Other experimental therapies targeted towards other CMT causal genes, like curcumin for myelin protein zero (MPZ; CMT1B) and HDAC6 inhibitors for heat shock protein 1 (HSPB1 mutants; CMT2F) were evaluated as well. ${ }^{22-24}$ Despite the promising data derived from these different CMT mouse models, no clinically successful treatment has so far been developed. This reflects the challenges in successfully translating novel therapeutics from mouse models to clinical practice and encourages us to contemplate about possible causes. In the next sections, we will cover the most studied relevant murine models for preclinical CMT research and critically discuss their relevance for preclinical therapeutic development.

\section{CMT1A PRECLINICAL STUDIES}

CMT1A caused by a $1.4 \mathrm{Mb}$ recurrent duplication of PMP22 represents $70 \%-80 \%$ of all CMT1 cases ${ }^{25}$ and therefore it is the most extensively studied CMT type. Different in vivo transgenic rodent models revealed that overexpression of this gene leads to hypomyelination/demyelination in Schwann cells and the extent of severity lies on the gene dosage. ${ }^{26-28}$ Immediately after, efforts were undertaken to reduce PMP22 expression with ascorbic acid (vitamin C) and progesterone antagonists. Ascorbic acid treatment was shown to ameliorate the peripheral neuropathy phenotype by reducing the PMP22 expression and consequently remyelinating the axons in the sciatic nerve of the C22 mouse overexpressing the human PMP22 gene. ${ }^{18}$ However, it failed at clinical trials. ${ }^{29}{ }^{30}$ Surprisingly, it was later identified that levels of PMP22 on the intact myelin were highly variable among patients with CMT1A as measured by immune-electron microscopy, and there was no correlation between disease severity, as measured by the CMT Neuropathy Score (CMTNS) and PMP22 expression levels. ${ }^{31}$ Concomitantly, the levels of PMP22 remained unaltered on ascorbic acid treatment. ${ }^{32}$ These observations suggested that PMP22 levels fluctuate over time and challenges the PMP22 dosage mechanism.

One of the possible reasons behind the failure of targeting PMP22 dosage as a mechanism might be the 'imperfect' modelling of the disease by rodent models of CMT1A. All these models were generated by a random insertion of multiple copies of PMP22 human CDNA, as insertion of the duplication of 1.4 Mb DNA segment was not possible. Thus, these models may not accurately reflect the altered genome or fluctuating levels of PMP22 in Schwann cells. This encouraged researchers to investigate the upstream pathomechanism leading to the altered homeostasis of PMP22 expression. ${ }^{33}$

Fledrich et al reported perturbed Schwann cell differentiation as one potential mechanism caused by an imbalanced activity of the PI3K-Akt and the Mek-Erk signalling pathways. They further demonstrated that early treatment of CMT1A rats with neuregulin 1 (NRG1; PI3K activator) prevents axonal loss and induces long-term improvement. ${ }^{34}$ However, a recent study claims that different isoforms of soluble NRG1 are upregulated in CMT1A nerves, questioning the NRG1 based therapeutic approach. ${ }^{35}$

Chumakov et al then reported PXT3003 (a combination of baclofen, naltrexon and D-sorbitol that act by reducing the levels of intracellular cAMP) through a systems biology targeting approach, with the aim to lower toxic PMP22 gene 
overexpression. ${ }^{36}$ Of note, this study used the same transgenic rat model as from the study of Fledrich et al and mainly targeted PMP22 expression as the therapeutic strategy. Based on positive results from this preclinical study and promising phase II data, PXT3003 is currently undergoing a phase III clinical trial. ${ }^{37}$

Recently, an antisense oligonucleotide (ASO) approach therapeutically targeting PMP22 RNA was shown to give excellent results in the two different rodent models of CMT1A. ${ }^{38}$ Mechanistically, there were dose-dependent increases in the mRNA levels of several lipid biosynthetic and myelin genes and decrease in dedifferentiation markers and myelin inhibitor, following 9 weeks of ASO treatment. Furthermore, they also demonstrated reduction in the level of rat PMP22 mRNA in the footpad suggesting its use as an outcome measure for this treatment. ${ }^{38}$

Overall, the therapeutic strategy in CMT1A still aims at reducing PMP22 expression using the same well-established rodent models. As a cautionary note, if PMP22 expression is reduced below its normal value, it can result in the other spectrum phenotype known as hereditary neuropathy with liability to pressure palsies (HNPP). At the pathological levels, the CMT1A duplication and HNPP deletion are well-characterised neuropathic entities but this may not be the case at the molecular level. The upcoming results from ongoing clinical trials will shed light on whether these rodent models are suitable models for CMT1A or whether just aiming at reducing PMP22 levels is sufficient for designing successful therapeutics. Keeping these conflicts in mind, a strategy involving molecular characterisation of the underlying deficits using a combination of disease models to identify stronger and clinically relevant targets should improve clinical success.

\section{PRECLINICAL STUDIES OF OTHER CMT SUBTYPES}

Protein misfolding is implicated in the pathogenesis of several neurological disorders including CMT. Two CMT1B mouse models with Arg98Cys and Ser63Del MPZ mutations, and the Pmp22 Trember I mice (a model for CMT1A but caused by a point mutation in PMP22), demonstrated activation of the unfolded protein response (UPR) as one of the mechanisms causing the peripheral neuropathy. ${ }^{39} 40$ As a result, treatment with curcumin and its derivatives (phosphatidylcholine curcumin designed to increase bioavailability and curcumin dissolved in sesame oil) improved the $M p z^{\mathrm{R} 98 \mathrm{C}}$ mice and Pmp22TrJ clinically, neurophysiologically and phenotypically by attenuating the UPR. Surprisingly, though CMT1B mutants as well as both Pmp22TrJ and Pmp22Tr variants are ER retained, UPR activation is not reported for Pmp22Tr animals. Nevertheless, the chances of clinical success for curcumin remain limited because of its instability and inefficient pharmacokinetics properties.

On the same line, Sephin1 (IFB-088) emerged as a candidate molecule with a promising pharmacokinetic profile for selectively inhibiting PPP1R15A (GADD34) involved in the UPR. ${ }^{s 1}$ On administering Sephin 1 orally to the transgenic $M p z^{\mathrm{S} 63 \mathrm{del}}$ mice modelling CMT1B, molecular (reduced the levels of ER-stress markers), morphological (rescued myelin thickness) and motor defects of the $M p z^{\text {S63del }}$ mice were completely rescued. In 2015, the US Food and Drug Administration (FDA) granted orphan drug designation to the InFlectis BioScience drug candidate IFB-088, for the treatment of CMT1. It remains to be confirmed using suitable cellular/mouse models how well IFB-088 will perform in the clinical trials and whether it could be applied to treat other forms of CMT as defective UPR/ER stress response is expected to be common for many inherited neuropathies. ${ }^{\text {s2 }}$
In addition, ectopic $\mathrm{Na}_{\mathrm{v}} 1.8$ expression at the unmyelinated segments was suggested in experimental CMT mouse models, ${ }^{\mathrm{s} 3}$ and in sural nerve biopsy samples from a patient with a severe form of CMT1B. ${ }^{s 4}$ Therefore, Rosberg et al demonstrated that oral treatment with subtype-selective $\mathrm{Na}_{\mathrm{v}} 1.8$ blockers (C31) in $M p z^{-/-}$mice, a different model of CMT1B, could acutely improve motor performance and excitability. ${ }^{\mathrm{s} 5}$ However, the mechanistic association between $\mathrm{Na}_{\mathrm{v}} 1.8$ expression and demyelination remains unknown. It is suspected that altered firing patterns due to increasing excitability of $\mathrm{Na}_{\mathrm{v}} 1.8$ channels aggravate the ' $\mathrm{Na}^{+}$mediated axonal toxicity' in CMT, leading to axonal degeneration. ${ }^{\text {s6 }}$

Though the efforts to find successful treatment for CMT1 are countless, CMT2 did not attract much attention, mostly because of a limited number of patients and the vast genetic heterogeneity. The first breakthrough came in 2011 when d'Ydewalle et al reported that HDCA6 inhibitors via deacetylation of tubulin could correct the axonal transport deficits and thus rescue the CMT2 phenotype in symptomatic mutant HSPB1 mice. ${ }^{23}$ Interestingly, their group and Zhongying et al expanded this finding for treating transgenic glycyl-tRNA synthetase mice (Gars ${ }^{\mathrm{P} 234 \mathrm{KY} /+}$ and Gars $^{\mathrm{C} 201 \mathrm{R} /+}$ ) exhibiting axonal transport deficits. ${ }^{\mathrm{s}}$, s8 Zhongying et al propose that mutant Gars directly interacts with HDAC6 protein and overactivates HDAC6, and the intensity of interaction determines the extent of the decrease in $\alpha$-tubulin acetylation level. This binding was observed in brain tissue of Gars $^{\mathrm{P} 234 \mathrm{KY} /+}$ mice, peripheral blood mononuclear cells (PBMCs) of patient with CMT2D and mutant-transfected NSC-34 cell lines but not in their corresponding wild-type controls. Remarkably, in CMT2D mice $\left(\right.$ Gars $^{\mathrm{P} 234 \mathrm{KY} /+}$ ), a decrease in $\alpha$-tubulin acetylation was specifically detected in sciatic nerves (not in brain or PBMCs) consistent with the peripheral nerve selectivity of the disease. However, it is unclear why this differential binding is then also found in other tissues.

On the contrary, Benoy et al demonstrated that HDAC6 interacts with GARS and this interaction can be reversed by tubastatin A; however, there is no difference in the binding affinity between wild type or mutant protein. They did not explore further as to why mutant GARS can affect HDAC6 function (figure 1A). Moreover, the long-term effects of HDAC6 treatment as well as its impact on other cellular functions besides axonal transport ${ }^{\mathrm{s} 9}$ remains to be determined (figure $1 \mathrm{~B}$ ). Thus, although HDAC6 inhibitors show promising preclinical data for multiple axonal CMT subtypes, a thorough analysis of HDAC6 along with its long-term effects interpreted in conjunction with cell-based mechanistic studies or in vivo studies manipulating HDAC6 levels in mice, is necessary before moving to clinical trials. Nevertheless, it is still worthwhile to note that enhanced binding properties for GARS mutant proteins have also been reported for neurophilin-1 (Nrp-1) receptor, which in turn disrupts the interaction with vascular endothelial growth factor (VEGF). ${ }^{s 10}$ Consequently, in those mice, expression of VEGF improved the phenotype while deletion of Nrp-1 worsened it.

It is also important to note that these axonal transport defects were measured in terms of mitochondrial trafficking. It remains an unanswered question whether the deficits in axonal transport are the result of impaired mitochondria as described in a study on primary motor neurons expressing wild type and mutant Hsp27 by Kalmar et al, 2017. ${ }^{\text {s11 }}$ Recently, Rocha et al identified mitofusin (MFN2) agonists that can allosterically activate MFN2, promote mitochondrial fusion, ameliorate mitochondrial dysmotility and mitochondrial trafficking deficits in cultured mouse neurons containing $M f n 2^{\mathrm{R} 94 \mathrm{G}}$ and $M f n 2^{\mathrm{T} 105 \mathrm{M}}$ mutations. Furthermore, they demonstrated that 
A

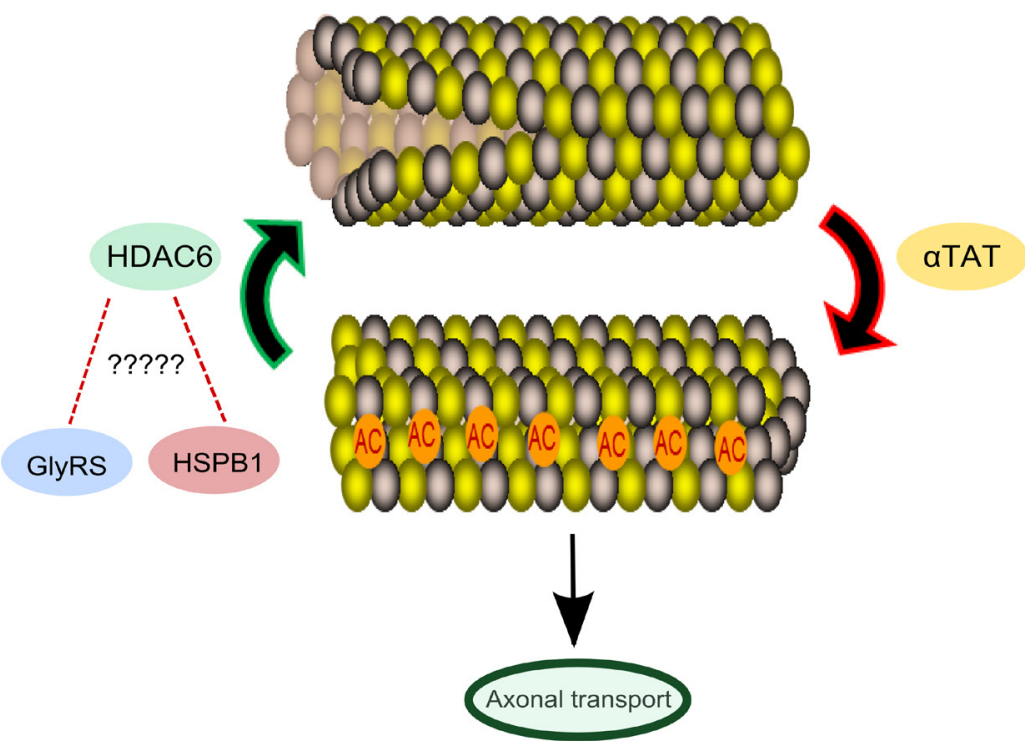

B

Figure 1 Schematic representation of tubulin acetylation and deacetylation. (A) CMT2-causal proteins like HSPB1 and GlyRS (GARS), through an unknown mechanism (direct or indirect interaction) regulate tubulin acetylation via HDAC6. However, the mutant proteins led to overactivation of the HDAC6 activity leading to reduced tubulin acetylation. So far, all the CMT2 preclinical studies involving HDAC6 inhibition uses rescue of axonal transport deficits as a read-out while the link to other molecular mechanisms remain missing. (B) HDAC6 has been involved in various molecular functions pertaining to neurodegeneration.

mitochondrial motility was restored to normal in the sciatic nerves of mice expressing the CMT2A mutant MFN2_T105M on treatment with the agonist. ${ }^{\text {s12 }}$ It would remain interesting to explore whether these agonists can thus improve the CMAP amplitudes which commensurate with the degree of axonal loss witnessed in patients with CMT2A and in these mouse models of CMT2.

Another possible strategy that emerged through several years of work in mouse models suggested a toxic gain-of-function of mutant proteins causing the phenotype. This has prompted many labs to opt for allele-specific knock-down or expression inhibition of causal genes using allele-specific oligonucleotides or RNAi-based gene therapy or small molecule inhibitors for a beneficial effect. This strategy is currently being tested on mouse models of CMT2D (GARS) and CMT2L (small heat shock protein $\mathrm{B} 8$; HSPB8).

As an alternative to all the above-mentioned approaches, Acceleron Pharma is searching for drugs that can improve the quality of life of patients by ameliorating the symptoms instead of targeting molecular pathways. ${ }^{\text {s13 }}$ They proposed a therapeutic strategy using ACE-083, focusing on improvement of foot dorsiflexor muscle function to prevent falls, thereby improving the neurogenic muscular atrophy. ACE-083 is a recombinant fusion protein that is a human follistatin linked to the human immunoglobulin G2 Fc domain. This protein inhibits myostatin activity and thus promotes skeletal muscle growth and differentiation. ${ }^{\text {s14 }}$ Importantly, testing on wild-type, mdx (Duchenne muscular dystrophy model), superoxide dismutase 1 (SOD1; amyotrophic lateral sclerosis (ALS) model) and Trembler-J mice (CMT) have provided positive results on muscle volume. ${ }^{\text {s15-18 }}$ Furthermore, a promising degree of muscle growth has been observed in the first-in human ACE-083 study with healthy volunteers. ${ }^{\text {s19 }}$ These studies support clinical development of ACE-083 owing to its wide applicability for various neuromuscular disorders, including CMT.

\section{DISEASE MODELLING OF CMT NEUROPATHIES USING IPSC MODELS: A FUTURE MODEL FOR PRECLINICAL AND PATHOMECHANISTIC STUDIES?}

It is evident that we still lack fundamental information on the pathophysiological processes that occur during the asymptomatic phase of the disease and the underlying molecular mechanisms that eventually lead to CMT. The traditional research models we have used for many years, such as mouse models and patient-derived lymphoblasts or fibroblasts, have important genetic and/or physiological limitations. Reprogramming of patient-derived somatic cells to induced pluripotent stem cells (iPSCs) has revolutionised the field of modern medicine. The development of iPSC technology brought with itself the promise to address the gap between pathogenesis and in vivo phenotypes. Neurological disorders benefit the most from the iPSC-disease modelling as it enables generation of disease-relevant cell types in vitro such as postmitotic neurons and glial cells, otherwise only available from sensory nerve biopsies and postmortem samples. Patient-specific iPSC-derived neural cells recapitulate the genotype and phenotypes of the diseases and can be manipulated like any other in vitro cellular model. Thus, they have the potential to considerably enrich our understanding of the pathogenesis, disease mechanism and may represent an appropriate model for drug screenings. This model has proven to be successful for studying several neurodegenerative diseases including frontotemporal dementia, Alzheimer's, Parkinson, Huntington disease, spinal muscular atrophy, ALS, Duchenne muscular dystrophy, schizophrenia and autism spectrum disorders ${ }^{\text {s20 }}$ and recently in CMT. $^{\text {21-22 }}$ In the next section, we will review this approach for CMT and discuss its limitations and potential for developing rational treatment strategies.

The first and foremost challenge with this technology is the establishment of reproducible, efficient and not so lengthy protocols for differentiating iPSCs into spinal motor neuron $(\mathrm{sMN})$ cultures. CMT research with the iPSC-derived sMN is 
currently at a naïve state. So far, most of the research is restricted to the phenotyping and validation of these iPSC-derived sMN as an appropriate model (table 2). The first study dates back to 2015, where Saporta et al generated sMN lines from patients with CMT2E (neurofilament; NEFL mutation) and CMT2A (Mitofusin; MFN2 mutation). ${ }^{\text {s21 }}$ They demonstrated an accumulation of intermediate filaments in the cell body of CMT2E motor neuron line, which was in line with the spinal cord motor neurons of heterozygous mice expressing the same point mutation in the NEFL gene. Additionally, they observed reduced action potential threshold and abnormal channel current properties that correspond to the patient phenotype. Their results were encouraging enough for using these iPSC-derived models as a human platform to study inherited neuropathies. However, they used a magnetic bead-based enrichment for L1CAM, a neuronal cell adhesion molecule associated with axon guidance and cell migration, for sorting out neuronal cells from the non-neuronal cells as their protocol could not provide pure motor neuron population. Fortunately, at this moment, differentiation protocols have been established to successfully generate sMNs with efficiency of up to $95 \% .{ }^{\text {s23 }}$ However, one cannot neglect the presence of contaminating mitotic progenitor populations in these highly efficient protocols and thus sorting pure motor neuron populations for global gene expression analysis may help to achieve more reliable results.

In 2016, Yon-Kim et al created iPSC lines from patients with CMT2F and dHMN2B, both caused by HSPB1 mutations, and differentiated these into motor neurons according to the method described by Amoroso et al. ${ }^{\text {s24 }}$ Consistent to the in vivo data, patient-derived motor neurons showed reduced absolute mitochondrial axonal trafficking velocity, percentage of moving mitochondria and tubulin acetylation compared with controls. Moreover, HDAC inhibitors were able to rescue axonal transport deficits in these iPSC-derived motor neurons via increasing the tubulin acetylation as shown by in vivo data. ${ }^{\mathrm{s} 25}$ It would be interesting to explore in future the molecular mechanism linking HSPB1 mutations with HDACs and its contribution towards axonal degeneration.

Deficits in autophagy have also emerged as a potential pathomechanism causing peripheral neuropathies. ${ }^{\text {s26 }}$ Interestingly, our group could demonstrate that autophagy is disrupted (decrease LC3II levels on bafilomycin treatment) in motor neurons differentiated from CMT2F/dHMN2B patient-derived iPSC (Haidar et al 2018, under review), suggesting novel therapeutic avenues. Additionally, one of our recent studies identified the actin-binding protein profilin2 (PFN2) as a common molecular determinant of CMT2 using proteomics on patient-derived lymphoblast lines. ${ }^{\text {s22 }}$ We could confirm these findings in CMT2A and CMT2E patient iPSC-derived motor neurons and in CMT2A and CMT2L mouse models. Our results on patient-derived sMN suggested the role for PFN2 in the pathogenesis of axonal CMT, also opening up the possibilities of its use as a potential biomarkers of disease.

Despite the preclinical success for treatment of CMT1A using different strategies in the rodent models of disease (PMP22 duplication, ASO, Ubiquitin pathway), the physiological consequences caused by PMP22 duplication or mutant PMP22 on Schwann cell development and/or myelin sheath formation, remain largely unknown. Recently iPSC models were evaluated for CMT1 as well. ${ }^{\text {s27-29 }}$ The major challenge behind using iPSC for studying CMT1 is the unavailability of a protocol that can differentiate iPSC into a fully functional myelinating Schwann cells. Though protocols have been published to differentiate Schwann cells from iPSC-derived NCSCs (neural crest stem cells), these protocols generally yield rather impure cultures and require months to generate functional Schwann cells. ${ }^{\text {s0-32 }}$ Despite the enrichment method using fluorescence-activated cell sorting or the 'LSB-short' method (named after the two inhibitors LDN-193189 and SB431542) based on dual SMAD inhibition, which demonstrates improved yields of NCSCs and a shorter differentiation time ( 8 days $)^{\mathrm{s} 3}$, a differentiation time of more than 40 days is still required to produce Schwann cells from NCSCs. ${ }^{\text {s34 }}$ Besides functionality, little is known regarding their effectiveness for axonal regeneration and recovery from peripheral nerve injury in vivo. Last year, Kim et al reported a method to generate fully functional Schwann cells precursors in 18 days followed by their differentiation into mature Schwann cells in one week. ${ }^{\mathrm{s} 35}$

Nevertheless, research groups have generated CMT1-iPSCs and differentiated these cells into p75 and human natural killer-1 (HNK-1) positive Schwann cells as obtained by FACS sorting. Kitani-Morii et al used iPSC derived NCSCs from patients with a genetically known demyelinating type of CMT neuropathy (CMT1A, CMT1B, CMT1D), for a global gene expression analysis. They found glutathione-mediated detoxification pathway as a molecular signature pathway common to these demyelinating subtypes. ${ }^{\text {s29 }}$ Remarkably, another study from Shi et al reported that differentiation of CMT1A iPSCs to Schwann cells was interrupted and instead gave rise to endoneurial fibroblast-like cells through NCSCs, unlike the normal control cells. Notably, ectopic PMP22-overexpression in control iPSCs recapitulates the aberrant Schwann cell differentiation of CMT1A. Furthermore, RNA sequencing measuring global expression profile of different developmental stages among iPSCs, the NCSCs and Schwann cells further confirmed the results. ${ }^{\text {s28 }}$ However, the expression of GSTT2 (glutathione S-transferase theta 2), the key player of glutathione detoxification pathway as described by the prior study was not described in this study.

\section{COMPARISON OF RESULTS FROM MOUSE MODELS WITH IPSCS}

The iPSC technology has not yet been exploited widely in the CMT research field. There are as of now, only a handful of studies, mostly characterising these iPSC-derived sMNs. These seminal studies clearly indicate that iPSC-derived sMN can recapitulate key disease-related features previously described in animal and cell-based models. Nevertheless, at the mechanistic level, the two models show both similarities and discrepancies that we would like to discuss in this section.

Differentially regulated transcripts have been explored in both NCSCs from a patient with CMT1A and from the sciatic nerve of PMP22 overexpressing rats. ${ }^{\mathrm{s} 28, \mathrm{~s} 36}$ No clear-cut similarities could be deduced from these high-throughput data. The key result from RNA sequencing of the rat model demonstrated a significant downregulation of the Cntf gene in PMP22 overexpressing rats pointing towards an axonal atrophy (the inability of Schwann cells to offer a trophic support to the axon). In addition, the CMT1A rats displayed a large group of downregulated genes involved in lipidic and glucidic metabolism, cytoskeleton and extracellular matrix components such as collagens. On the other hand, NCSCs from the patient with CMT1A showed an upregulation of genes involved in fibrogenesis, autophagy, formation of actin filaments and formation of actin stress fibres in addition to the reduced profile related to cholesterol synthesis and lipid metabolism. ${ }^{\text {s2 }}$ Interestingly, most of these differentially regulated apolipoprotein proteins have previously been shown to be related to myelin biosynthesis and/or demyelination. ${ }^{\mathrm{s} 7-41}$ 
岕芯
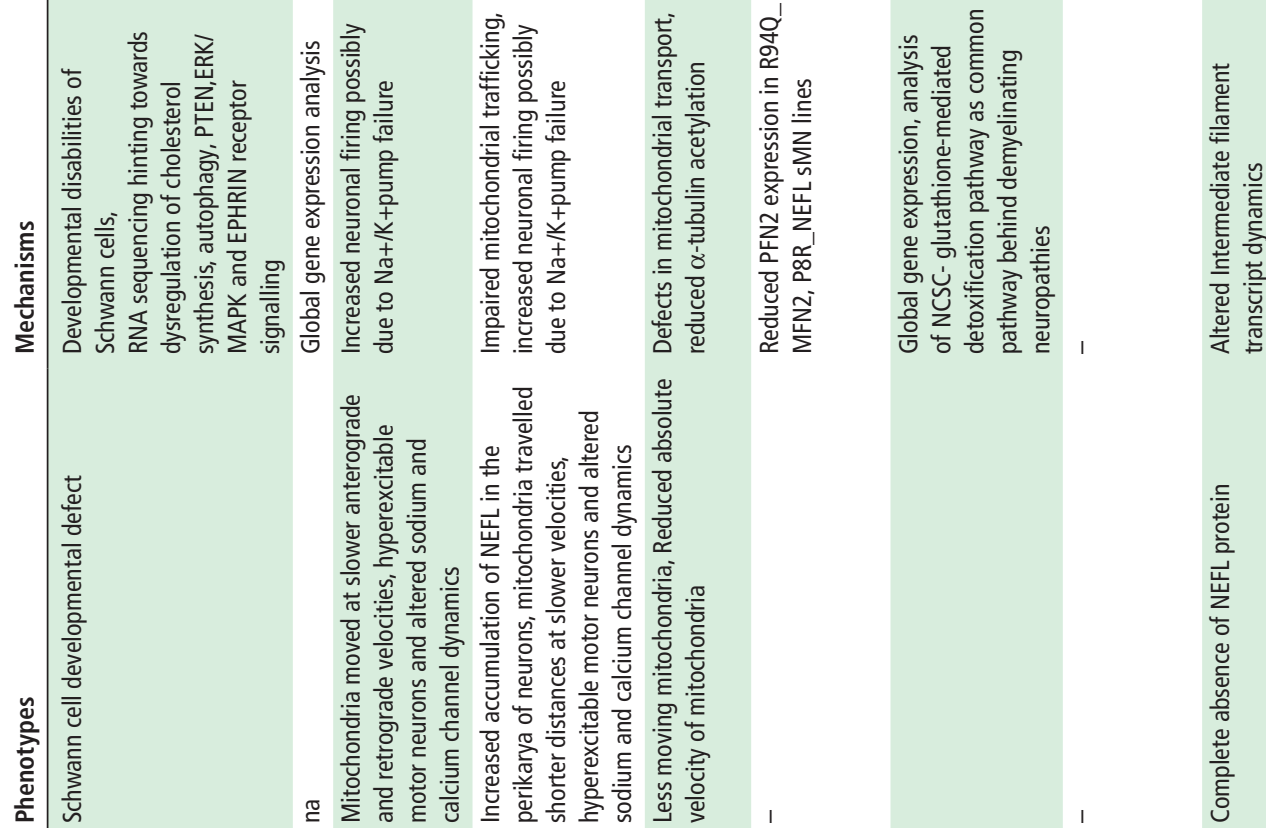

흠

离

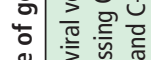

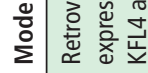

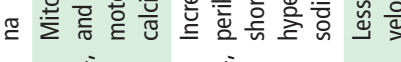

兑

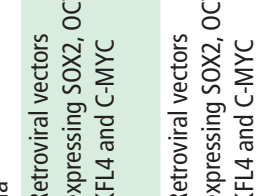

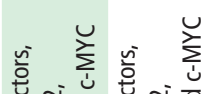

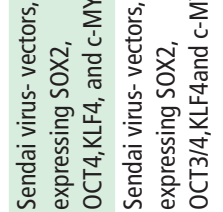

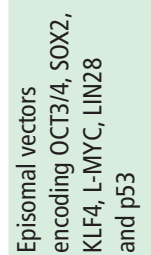

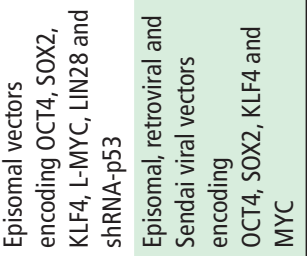

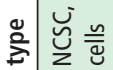

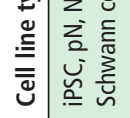

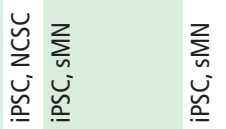

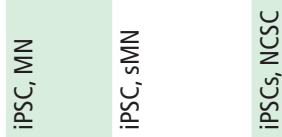

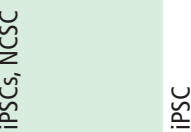

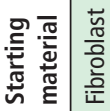

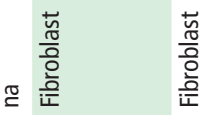

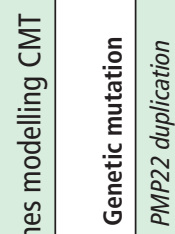

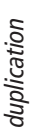

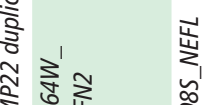

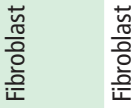

$\frac{\sqrt{0}}{\frac{\pi}{0}}$

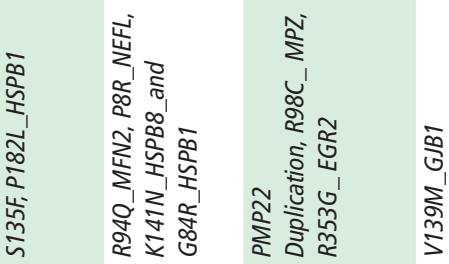

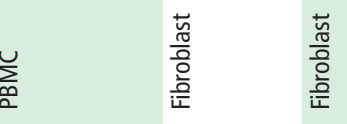

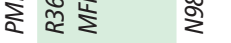

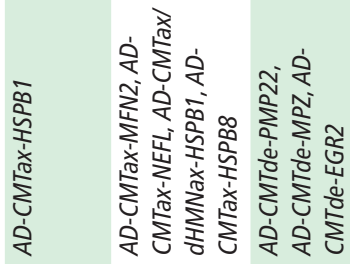
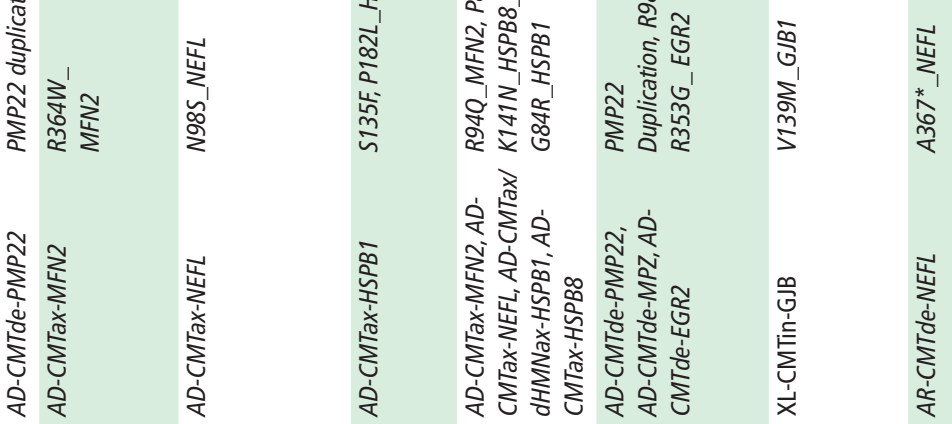
In addition, statistically significant canonical pathways using IPA (Ingenuity Pathway Analysis) were identified that included RhoGDI, PTEN, ERK/MAPK, mTOR and EPHRIN receptor signalling pathways in CMT1A cells, among others. Intriguingly, both in CMT1A rats and in the primary Schwann cell cultures derived from these animals, MEK-ERK signalling was found to be upregulated. Hyperactivation of MEK1/2-ERK1/2 causing MCP-1/CCL2 upregulation has already been described causing a CMT phenotype in Pmp22 transgenic mice. ${ }^{.42}$ Fledrich et al further reported a reduced activity of PI3K-Akt signalling contributing towards the hyperactivation of the MEK-ERK pathway in CMT rats. Their group demonstrated that early neuregulin-1 (NRG1) therapy in transgenic rats induced the PI3K-Akt activation and restored the disturbed ratio of PI3K-Akt and MEK-ERK signalling. This led to reduction of the dedifferentiation and immaturity markers and eventually improved differentiation of Schwann cells and myelination of axons. ${ }^{34}$ On the contrary, Schwann cells obtained from iPSC lineages indeed showed a higher ERK activation, though no changes in PI3K-AKT phosphorylation levels were observed in CMT1A/ PMP22 lines compared with control. ${ }^{\text {s28 }}$ Overall, the data from both animal models and iPSC lines does highlight the significance of the MEK-ERK activation in causing Schwann cell differentiation deficits.

For CMT2 models, ultrastructural analysis of spinal cord and sciatic nerves of 1 -year-old knock-in Nefl ${ }^{\mathrm{N} 985}$ mice revealed that the neurofilaments were primarily accumulated in the cell bodies of the neurons, where they formed aggregates. Furthermore, the predominant structures in the nerves of the $\mathrm{Nefl}{ }^{\mathrm{N} 98 \mathrm{~S} /+}$ mouse were microtubules rather than the abundant neurofilaments that can be observed in the $\mathrm{Nefl}^{+/+}$mice. ${ }^{\text {s43 }}$ These results were in agreement with the immunohistochemistry of iPSC-derived neurons showing an increased accumulation of NEFL in the perikarya of neurons from two distinct clones. ${ }^{\text {s21 }}$

Furthermore, abnormal mitochondrial trafficking has been implicated as a part of the pathophysiology of CMT2A. The animal models of CMT2A demonstrate a differential mitochondrial phenotype compared with wild type littermates (altered number of mitochondria, uneven distribution of mitochondria, aggregated mitochondria clustered around the axon, more mitochondria that spend time paused, anterograde and retrograde velocity alterations and so on). ${ }^{544-47}$ Whereas the CMT2A patient-derived sMN neurons did not demonstrate such severe mitochondrial deficits, the study from Saporta et al suggested slower anterograde and retrograde velocities of mitochondria in patient lines when compared with the controls. ${ }^{\text {s21 }}$

Also interesting is to note that sMNs derived from iPSCs carrying a mutation in HSPB1 demonstrated reduced absolute velocity and percentage of moving mitochondria in axons for both S135F and P182L mutants compared with controls. In case of DRG neurons isolated from symptomatic HSPB1_S135F expressing mice, fewer moving mitochondria were observed along with a reduction of total number of mitochondria instead of total reduced velocities. Of note, both models clearly indicated decrease in tubulin acetylation as a possible cause of mitochondrial deficits, which were then restored by HDAC6 inhibitors. However, DRG neurons from mice expressing HSPB1_P182L did not show any alteration in mitochondrial dynamics at all. ${ }^{23, \mathrm{~s} 25}$

\section{LESSONS LEARNT FROM ANIMAL MODEL: PHYSIOLOGICAL,} GENETIC AND TECHNICAL LIMITATIONS

Despite the very high rate of preclinical success, clinical success is yet to be achieved despite 25 years of intensive investigation.
This suggests possible deficiencies in our current understanding of the disease. For instance, the most studied CMT1A subtype is caused by a large segmental duplication of $1.4 \mathrm{Mb}$ containing PMP22 plus eight other genes. It is difficult for any cloning method to recapitulate the same genetic alteration. Since an early study on human sural nerve biopsy revealed increased levels of PMP22 in patients ${ }^{\mathrm{s} 48}$, it formed the rationale behind all animal model development and therapeutic intervention strategies so far. There have been mounting evidences in the last few years of research suggesting that PMP22 levels are extremely variable, they fluctuate among patients and may fall into normal ranges at times. ${ }^{31,33, s 49}$ Thus, the current CMT1A models need to be carefully scrutinised before conducting preclinical studies focusing primarily on the suppression of PMP22 expression to avoid being a prey of more failed clinical trials. ${ }^{33}$

The technical limitation that comes with the animal models is the difference in physiology compared with humans and the expression pattern of the gene in question. As it can be appreciated from table 1 , most of the animal models generated are transgenic models overexpressing the mutant proteins, usually a human transgene. In some models, the expression is rather restricted to the nervous system by specific promoters while in others the transgene expression is ubiquitous. The question remains as whether a physiological level of the mutant protein is necessary to adequately understand the biology behind the disease. Will the humanised mice with a human transgene randomly integrated into the mouse genome have the same regulatory mechanism as in the patients? Can overexpression models adequately mimic the disease pathology? Another point to consider is that many of these studies made use of knock-out (KO) mice to model a loss-of-function mutation. One could question whether a loss-ofknown function or an unknown gain-of-function is responsible for pathogenicity. Due to advances in genetic engineering, it has now become easier to generate knock-in (KI) mice models with physiological expression level of the causal gene and mutant protein. Our group has been able to successfully generate $H s p b 8$ $\mathrm{KI}$ and $\mathrm{KO}$ mouse models of which the KI closely mimics the CMT2L/dHMN patient phenotype. Mechanistically, these Hspb8 KI/KO mouse uncovered a novel gain-of-toxic function that is contributing to the phenotype instead of a loss-of-function, as the KO mice appeared normal during the follow-up time of 12 months. However, it is critical to note that despite being an autosomal dominant disease, the motor deficits were only observed in the homozygous $H s p b 8^{K 141 N / K 141 N}$ animals. The heterozygous $H s p b 8^{K 141 N /+}$ mice with an allelic pattern similar to the patient failed to develop a disease phenotype. ${ }^{\text {s50 }}$ This need of higher expression of mutant protein to reproduce a functional phenotype might be attributed to the discrepancies between mice and humans in terms of their respective life span, the disease duration and exposure to different environment. ${ }^{s 47}$

Furthermore, Adebola et al reported two different KI mouse models with mutations in Nefl linked to CMT2E $\left(\mathrm{Nefl}^{\mathrm{P} 8 \mathrm{R} /+}\right.$, $\mathrm{Nefl}^{\mathrm{PBR} / \mathrm{P8R}}$, Nefl $\left.{ }^{\mathrm{N98S/+}}\right)$. While both the Nefl $\mathrm{PRR}^{\mathrm{PR}+}$ and Nefl ${ }^{\mathrm{P8R} /}$ ${ }^{\mathrm{P} 8 \mathrm{R}}$ KI mice failed to demonstrate a disease phenotype with the batteries of assays performed by the group, $\mathrm{Nefl}^{\mathrm{N98S} /+}$ mice (which is an early onset neuropathy) were symptomatic starting as early as postnatal day 7 . It was surprising to observe no abnormalities in the Nefl ${ }^{\mathrm{P} 8 \mathrm{R} /+}$ and Nefl ${ }^{\mathrm{P} 8 \mathrm{R} / \mathrm{P} 8 \mathrm{R}}$ mice especially given that this mutation causes a severe form of neuropathy in patients. Another astonishing fact was that $\mathrm{Nefl}^{\mathrm{N} 98 \mathrm{~S} /+}$ mice displayed no disease progression and severity on aging. ${ }^{543}$

Does such a finding suggest that the short lifespan of mice is inadequate to model late onset neuropathy? In any case, the enigma remains as if why in some mice, symptoms could be 
seen quite early while in others it takes more than a year, and still some mice live disease-free despite carrying a pathogenic mutation. It is also possible that these so-called pathogenic mutations may act synergistically with other unknown genetic and epigenetic modifiers that could potentially accelerate or delay the phenotype.

\section{CONSIDERATIONS BEFORE USING IPSC MODELS AND FUTURE PERSPECTIVES}

Despite all the concerns with the animal models, there is a positive side to consider. Clinicians are now systematically collecting patient-derived biomaterials, blood or skin biopsies thereby ensuring continuous supply for fibroblasts and lymphoblasts lines from patients with CMT. These are valuable resources for genetic exploration and for in vitro disease modelling by reprogramming them into iPSC lines. Though the data available so far from these models are preliminary, we anticipate that this in vitro system would facilitate mechanistic understanding, target identification as well as drug screening. Kim et al recently validated the synergistic applicability of this disease model along with an animal model, where they positively assessed the therapeutic effect of HDAC6 inhibitors using motor neurons derived from iPSCs of patients with CMT2F and dHMN2B. ${ }^{\mathrm{s} 25}$ Their data highlight the possibility for the use of iPSC-derived lines for drug screening and for future mechanistic studies in the field of CMT.

However, we must not ignore that like all other models, iPSC models have their own shortcomings. The major limitation with iPSC modelling is the variability issue. This is mostly because this field - up until recently-was in its experimental phase. Researchers across the world have been optimising protocols for reprogramming and differentiation. Thus, at this point when no gold standard has been defined for this technology, naturally, it has led to reproducibility issues. Looking at the data we currently have, we can already highlight the variability among different labs in terms of (1) starting material used for reprogramming; (2) in reprogramming protocols; (3) in selecting the best iPSC clones; (4) in differentiation protocols; (5) in subpopulations obtained during differentiations; (5) variation in controls used to compare the data: isogenic, derived in the same way, age-matched and gender-matched; (6) interclonal variability and possibly more. Hence, there remains an utmost need to define the benchmarks or gold-standards for this technology to generate data that is comparable and biologically relevant. Besides the variability issue, these iPSC models have other limitations that need to be taken into account when using them to model peripheral neuropathies, for example: (1) immature state of differentiated cells for modelling late-onset disease, (2) lack of axo-glial and systemic interactions, (3) lack of neuromuscular junction formation and modelling. Moreover, it remains to be shown how iPSC-derived motor neurons from patients with CMT can efficiently probe degenerating axonal phenotypes and deal with the length-dependent nature of the disease compared with control lines.

Our goal with this review was to highlight the limitations of the currently used research models and design better experiments that overcome the numerous challenges. We believe that it is time to move beyond the 'Rat race'. Instead, as researchers we need to accept that it is a prerequisite to validate our hypotheses in human-like models before taking the results further into clinical trials. The use of animal models remains indispensable for translational research, but we can strengthen it by synergising with accurately generated iPSC derived motor or sensory neurons from patients with CMT. Nonetheless, caution is advisable for selecting the correct rodent model for the specific CMT subtype.

Additional references can be found in the online supplementary material 1.

Contributors MJ, JB, MAS and VT contributed to the writing of this review.

Funding The research by MJ and VT are supported in part by the Fund for Scientific Research (FWO-Flanders), the 'Association Belge contre les Maladies Neuromusculaires' (ABMM), the Medical Foundation Queen Elisabeth (GSKE), the Association Française contre les Myopathies (AFM), the Muscular Dystrophy Association (MDA) and the Solve-RD project from the European Union's Horizon 2020 research and innovation programme under grant agreement № 779257.

Competing interests None declared.

Patient consent Not required.

Provenance and peer review Not commissioned; externally peer reviewed. Data sharing statement No additional data are available.

Open access This is an open access article distributed in accordance with the Creative Commons Attribution Non Commercial (CC BY-NC 4.0) license, which permits others to distribute, remix, adapt, build upon this work non-commercially, and license their derivative works on different terms, provided the original work is properly cited, appropriate credit is given, any changes made indicated, and the use is non-commercial. See: http://creativecommons.org/licenses/by-nc/4.0/

\section{REFERENCES}

1 Barreto LC, Oliveira FS, Nunes PS, et al. Epidemiologic study of Charcot-Marie-Tooth disease: a systematic review. Neuroepidemiology 2016:46:157-65.

2 Tooth $\mathrm{HH}$. The peroneal type of progressive muscular atrophy [Thesis]. London: University of London, 1886.

3 Charcot JM, Marie P. Sur une forme particulière d'atrophie musculaire progressive souvent familiale débutant par les pieds et les jambes et atteignant plus tard les mains. 1886

4 Timmerman V, Strickland AV, Züchner S. Genetics of Charcot-Marie-Tooth (CMT) disease within the frame of the human genome project success. Genes 2014;5:13-32

5 Barisic N, Claeys KG, Sirotković-Skerlev M, et al. Charcot-Marie-Tooth disease: a clinico-genetic confrontation. Ann Hum Genet 2008;72:416-41.

6 Kennedy RA, Carroll K, McGinley JL. Gait in children and adolescents with CharcotMarie-Tooth disease: a systematic review. J Peripher Nerv Syst 2016;21:317-28.

7 Burns J, Ramchandren S, Ryan MM, et al. Determinants of reduced health-related quality of life in pediatric inherited neuropathies. Neurology 2010;75:726-31.

8 Redmond AC, Burns J, Ouvrier RA. Factors that influence health-related quality of life in Australian adults with Charcot-Marie-Tooth disease. Neuromuscul Disord 2008;18:619-25

9 Pareyson D, Scaioli V, Laurà M. Clinical and electrophysiological aspects of CharcotMarie-Tooth disease. Neuromolecular Med 2006:8:3-22.

10 Pareyson D, Saveri P, Pisciotta C. New developments in Charcot-Marie-Tooth neuropathy and related diseases. Curr Opin Neurol 2017;30:471-80.

11 Saghira C, Bis DM, Stanek D, et al. Variant pathogenicity evaluation in the community-driven Inherited Neuropathy Variant Browser. Hum Mutat 2018;39:635-42

12 Magy L, Mathis S, Le Masson G, et al. Updating the classification of inherited neuropathies: results of an international survey. Neurology 2018;90:e870-e876.

13 Weis J, Claeys KG, Roos A, et al. Towards a functional pathology of hereditary neuropathies. Acta Neuropathol 2017;133:493-515.

14 Wong $\mathrm{CH}$, Siah KW, Lo AW. Estimation of clinical trial success rates and related parameters. Biostatistics 2018.

15 Suter U, Welcher AA, Ozcelik T, et al. Trembler mouse carries a point mutation in a myelin gene. Nature 1992;356:241-4.

16 Martini R, Zielasek J, Toyka KV, et al. Protein zero (P0)-deficient mice show myelin degeneration in peripheral nerves characteristic of inherited human neuropathies. Nat Genet 1995;11:281-6.

17 Fledrich R, Stassart RM, Sereda MW. Murine therapeutic models for Charcot-MarieTooth (CMT) disease. Br Med Bull 2012;102:89-113.

18 Passage E, Norreel JC, Noack-Fraissignes P, et al. Ascorbic acid treatment corrects the phenotype of a mouse model of Charcot-Marie-Tooth disease. Nat Med 2004;10:396-401.

19 Meyer zu Horste G, Prukop T, Liebetanz D, et al. Antiprogesterone therapy uncouples axonal loss from demyelination in a transgenic rat model of CMT1A neuropathy. Ann Neurol 2007;61:61-72

20 Sereda MW, Meyer zu Hörste G, Suter U, et al. Therapeutic administration of progesterone antagonist in a model of Charcot-Marie-Tooth disease (CMT-1A). Nat Med 2003;9:1533-7 
21 Sahenk Z, Nagaraja HN, McCracken BS, et al. NT-3 promotes nerve regeneration and sensory improvement in CMT1A mouse models and in patients. Neurology 2005;65:681-9.

22 Khajavi M, Shiga K, Wiszniewski W, et al. Oral curcumin mitigates the clinical and neuropathologic phenotype of the Trembler-J mouse: a potential therapy for inherited neuropathy. Am J Hum Genet 2007;81:438-53.

23 d'Ydewalle C, Krishnan J, Chiheb DM, et al. HDAC6 inhibitors reverse axonal loss in a mouse model of mutant HSPB 1-induced Charcot-Marie-Tooth disease. Nat Med 2011;17:968-74.

24 Khajavi M, Inoue K, Wiszniewski W, et al. Curcumin treatment abrogates endoplasmic reticulum retention and aggregation-induced apoptosis associated with neuropathycausing myelin protein zero-truncating mutants. Am J Hum Genet 2005;77:841-50.

25 Bird TD. Charcot-Marie-Tooth Neuropathy X Type 1. In: Pagon RA, Adam MP, Ardinger HH, eds. GeneReviews(R). Seattle (WA), 1993.

26 Huxley C, Passage E, Manson A, et al. Construction of a mouse model of CharcotMarie-Tooth disease type 1A by pronuclear injection of human YAC DNA. Hum Mol Genet 1996;5:563-9.

27 Huxley C, Passage E, Robertson AM, et al. Correlation between varying levels of PMP22 expression and the degree of demyelination and reduction in nerve conduction velocity in transgenic mice. Hum Mol Genet 1998;7:449-58.

28 Sereda M, Griffiths I, Pühlhofer A, et al. A transgenic rat model of Charcot-MarieTooth disease. Neuron 1996;16:1049-60.

29 Pareyson D, Reilly MM, Schenone A, et al. Ascorbic acid in Charcot-Marie-Tooth disease type 1A (CMT-TRIAAL and CMT-TRAUK): a double-blind randomised trial. Lancet Neurol 2011;10:320-8.

30 Lewis RA, McDermott MP, Herrmann DN, et al. High-dosage ascorbic acid treatment in Charcot-Marie-Tooth disease type 1A: results of a randomized, double-masked, controlled trial. JAMA Neurol 2013;70:981-7.
31 Katona I, Wu X, Feely SM, et al. PMP22 expression in dermal nerve myelin from patients with CMT1A. Brain 2009;132:1734-40.

32 Nobbio L, Visigalli D, Radice D, et al. PMP22 messenger RNA levels in skin biopsies: testing the effectiveness of a Charcot-Marie-Tooth 1A biomarker. Brain 2014; 137:1614-20.

33 Li J. Caveats in the established understanding of CMT1A. Ann Clin Trans/ Neurol 2017;4:601-7

34 Fledrich R, Stassart RM, Klink A, et al. Soluble neuregulin-1 modulates disease pathogenesis in rodent models of Charcot-Marie-Tooth disease 1A. Nat Med 2014; 20:1055-61.

35 Fornasari BE, Ronchi G, Pascal D, et al. Soluble Neuregulin1 is strongly upregulated in the rat model of Charcot-Marie-Tooth $1 \mathrm{~A}$ disease. Exp Biol Med 2018:243:370-4.

36 Chumakov I, Milet A, Cholet N, et al. Polytherapy with a combination of three repurposed drugs (PXT3003) down-regulates Pmp22 over-expression and improves myelination, axonal and functional parameters in models of CMT1A neuropathy. Orphanet J Rare Dis 2014;9:201.

37 Attarian S, Vallat JM, Magy L, et al. An exploratory randomised double-blind and placebo-controlled phase 2 study of a combination of baclofen, naltrexone and sorbitol (PXT3003) in patients with Charcot-Marie-Tooth disease type 1A. Orphanet Rare Dis 2014;9:199.

38 Zhao HT, Damle S, Ikeda-Lee K, et al. PMP22 antisense oligonucleotides reverse Charcot-Marie-Tooth disease type 1A features in rodent models. J Clin Invest 2018;128:359-68.

39 Bai Y, Patzko A, Shy ME. Unfolded protein response, treatment and CMT1B. Rare Dis 2013:1:e24049.

40 Okamoto Y, Pehlivan D, Wiszniewski W, et al. Curcumin facilitates a transitory cellular stress response in Trembler-J mice. Hum Mol Genet 2013;22:4698-705. 